\title{
Characterization of a Passenger Vessel Sinking and Finally Foundering in Lacustrine Environment
}

\author{
Peter Oropeza, Diego Hurtado, Edgar Gil, Pablo Maldonado, \\ Miguel Ruiz \\ Polytechnic University of the State of Morelos, Morelos, Mexico \\ $\{$ mruizj\}@live.com.mx
}

\begin{abstract}
This research considers a simulating crowds into a passenger vessel Evacuation in the face of fire and the Effect of Human Behavior and Building Characteristics on Evacuation, the most crucial aspect of a passenger's vessel safety in the face of fire is the possibility of safe and efficient escape. The study of attitudes and behavior of passengers in relation to reactions to alarms, regulations, emergency signals, smoke and fire. An important precondition is that its fire safety facilities enable independent and adequate fire response performances by the passenger's vessel. In practice, it appears that the measures currently required by law do not always provide the support that people in burning cruiser's need and because of lack of signs. In such circumstances, timely and effective evacuation of all passengers and crew will be extremely important, and failure to evacuate in time may lead to catastrophic consequences. Consequently, understanding how individuals behave in the case of fire and fire evacuation is essential if we are to bring fire safety measures into line with occupants' needs during an incident. This paper contains a review of the available literature on human behavior in a fire so far as ship safety is concerned. The findings are presented as an overview of the critical factors which determine occupants' fire response performances, namely the characteristics of fire, human beings and ship.
\end{abstract}

Keywords: Multi-agent System, Fire response performance, Crowd Simulation, Evacuation, Human behavior under fire, Safety regulations.

\section{Introduction}

On the model of the passenger vessel related with evacuation in features face of fire using software simulation and the effect of human behavior and characteristics of a safe evacuation, The goal of this research is concerned that many of the actions taken by those responsible for the safety of passengers on a vessel are based on incorrect stipulations of how passengers will behave on an sinister in this case of fire aboard, Consequently the decision to raise the alarm and begin preliminary evacuation preparations is often delayed with resultant disastrous consequences. The different levels of organizations in case of an 
emergency are the level of operations, the level of communication and coordination, the level of design, the level of culture \& morality, and the level of social structures \& organizations [9]. In every case should result in reactive to improve safety and efficiency in form of implementing a system of finding the shortest paths between departures in vessel decks for each passenger using a bracelet and implementing and a signaling system using leds and lasers, depending of the case of the risk in the vessel. One of the reasons for this is that even though a good training and procedures are essential to safe and efficient operations, human error will only be minimized but no eliminated, we therefore need to improve the emergency departures and the organization culture depends of the latent cause of erroneous actions because of lacks of signals. "The 1995 amendments to the International Convention on Standards of Training, Certification and Watch keeping for Seafarers, 1978, (STCW 95)", outline specific requirements for safety-related training. Included in this training is the requirement that all persons having responsibility for the safety of passengers in an emergency on board a passenger vessel undertake training in crisis management and human behavior in emergencies. The factors that impacts is how the crew were trained and even the passengers training through exercises on board vessel, to design new corridors with some kind of lighting that can help the passengers to guide them to the departures and lasers depending of the danger of the corridor, leisure areas and accommodation space, and to implement of a safety plan to prevent disasters. The factors such as delayed reaction to alarms, the walking speed depending of the type of person, is different the walking speed of a adult of old man, the fact that usually every passenger try to find his family members before being evacuated and problems with instructions from the crew, lacks of signals, the language of the signals or even panic, these humans factors problems should be addressed as part of the evaluation of efficiency of evacuation. The others parts that the project carried out experimental studies of the safety and affiance of lighting corridors and emergency led signs and the efficiency of different techniques of evocation like lifeboats, lifejackets, fire suppression system of the vessel and slide and fall system [3]. Usually some passengers will refuse to leave a particular place where they had an agreement to meet again with relatives. Other passengers will refuse to go directly to the assembly station because they want to search the ship to find their children, spouse or other relatives. These parameters are taken into account in evacuation simulation models. When the fire occurs, the smoke reduces the visibility and it will put and added pressure on evacuation because of the reduced time available of a secure scape and the hard way to find the closest and safest departures, and the visibility. Under these circumstances panic can also arise, if passengers get trapped in closed areas or if they judge that there is enough time for orderly escape because of the reduced visibility and finding the shortest and secure paths between exits in vessel decks. Usually the vessels passengers are guided towards and verbally instructed to go to the nearest exit without delay and under these circumstances, evacuation paths are not an issue. Technical assistance for way finding by means of graphic and acoustic signs or through the architectural design becomes important in large buildings and passengers ship, where the spatial layout is more complex [4,5]. Here we find many different sings like maps and networks that are usually used to direct occupants and passengers under normal 
conditions of way finding. Way finding systems and acoustic sings as technical support for way finding during evacuation, especially in cases of smoke and in fire $[7,8]$.

\section{$2 \quad$ Methodology}

\subsection{Pedestrian Forces}

The forces that accelerate a pedestrian are the result of internal (will) and external (collision, signals, etc.) forces [13]. An alert individual will try to avoid collisions before they happen, resulting in vanishing external forces. However, if the pedestrian density increases, collision forces will appear and the ability to move freely will be impaired. The difference between external and internal forces is admittedly not too rigorous, and only serves descriptive purposes in the present context. The forces considered are:

Internal forces: Will force, Pedestrian collision avoidance forces and Obstacle/wall avoidance forces.

External forces: Pedestrian contact forces and Obstacle/wall contact forces.

Additionally, we consider: Kinematic constraints: Motion inhibition forces.

We denote by will force the force that will accelerate (or decelerate) a pedestrian to achieve the velocity it desires. Depends by factors such as height (H), weight (W), footwear (F), drunkenness (D), inability (I), fatigue (FA). Given a desired velocity vd and the current velocity $\mathrm{v}$, this force will be of the form:

$$
\mathrm{VT}=\text { age } * \text { displacement }(\mathrm{H}, \mathrm{W}, \mathrm{F}, \mathrm{D}, \mathrm{I}, \mathrm{FA}) .
$$

The modeling aspect is included in the function of the effects of "group binding" (finding family members before being evacuated), which we denote by produce force the force that will accelerate - decelerate a pedestrian or the crown to achieve the velocity it desires. Because some passengers will refuse to leave a particular place, where they had an agreement to meet again with relatives, other passengers will refuse to go directly to the assembly station because they want to search the ship to find their children, spouse or other relatives. We can thus obtain the speed of walking by measuring the time required to reach a percentage (e.g. 40\%) of the desired velocity, starting from rest. This speed is typically in the range of $17 \mathrm{~km}$ per hour but obviously depends on the pedestrian's current state of fitness and stress, fatigue, drunkenness, inability, group binding, footwear, weight, height, desire to reach a goal, climate, signals, and noise, among others.

In this case, we have an equation:

$$
v(t i)=\frac{E * K(t i) * \frac{\sum_{j=1}^{n} j}{n} \lambda}{C} \simeq 1,
$$


where

E: Age,

$\mathrm{K}$ : Levels of care,

$\mathrm{K}=(\mathrm{a}+\mathrm{b}) / 2$,

a: level of drunkenness,

b: level of fatigue,

$\mathrm{J}$ : attribute of the agent,

I: Agent,

t: Time,

$\mathrm{n}$ : number of attributes per agent,

$\lambda$ : inability,

C: Number of relatives.

\subsection{Sociocultural Model}

Sociocultural model is defined as:

$$
\begin{aligned}
& \text { F reaction } \square=\left(\mathrm{P} * \lambda * \mathrm{~T}^{*} \mathrm{U} * \mathrm{~V} * \mathrm{~A}(\mathrm{x})\right) \rrbracket^{\wedge} \mathrm{s}, \\
& \lambda=(\mathrm{q}+\mathrm{n}) / 2,
\end{aligned}
$$

where:

Religiosity $=\mathrm{P}$,

Education level $=\mathrm{Q}$,

Education $=\lambda$,

Values $=\mathrm{R}$,

Language $=\mathrm{S}$,

Social level $=\mathrm{T}$,

Region $=\mathrm{V}$,

I: The universe of existing languages,

$\mathrm{S}$ : The set of languages that dominates the person,

$\mathrm{A}(\mathrm{x})$ : The person dominates the language the native language of the vessel, $\mathrm{K}=$ Optimal Value,

$\mathrm{S}=$ Level of solidarity.

\subsection{The Shortest and Secure Path}

We use graphs to find the shortest paths or routes from starting point to a final destination between departures in vessel decks, in order to represent the shortest path, we use graphs. A graph is mathematical abstract object, to represent the way of the passenger in the vessel, we use vertices and edges, which Edges connects pairs of vertices in this case exits. Where vertices represent exits and edges represent routes that connect the exits. Along the routes of the graph it is possible to walk by moving from one vertex to other vertices. Depending 
on whether or not one can walk along the routes by both sides or by only one side determines if the graph is a directed graph or an undirected graph, in this case is a directed graph. In addition, lengths of routes are called weights, and the weights are used for calculating the shortest path from routes point to another routes. There exist different types of algorithms that solve the shortest path problem. However, only several of the most popular conventional shortest path algorithms adapts to our model and is based in Dijkstra's Algorithm [10]. The algorithm's that are going to be discussed in this paper: Dijkstra's Algorithm, Floyd-Warshall Algorithm, Bellman-Ford Algorithm and Genetic Algorithm. We proposed an algorithm obtained by extending the Dijkstra's algorithm [10]. In this paper we use it for solving the single source shortest path problem.
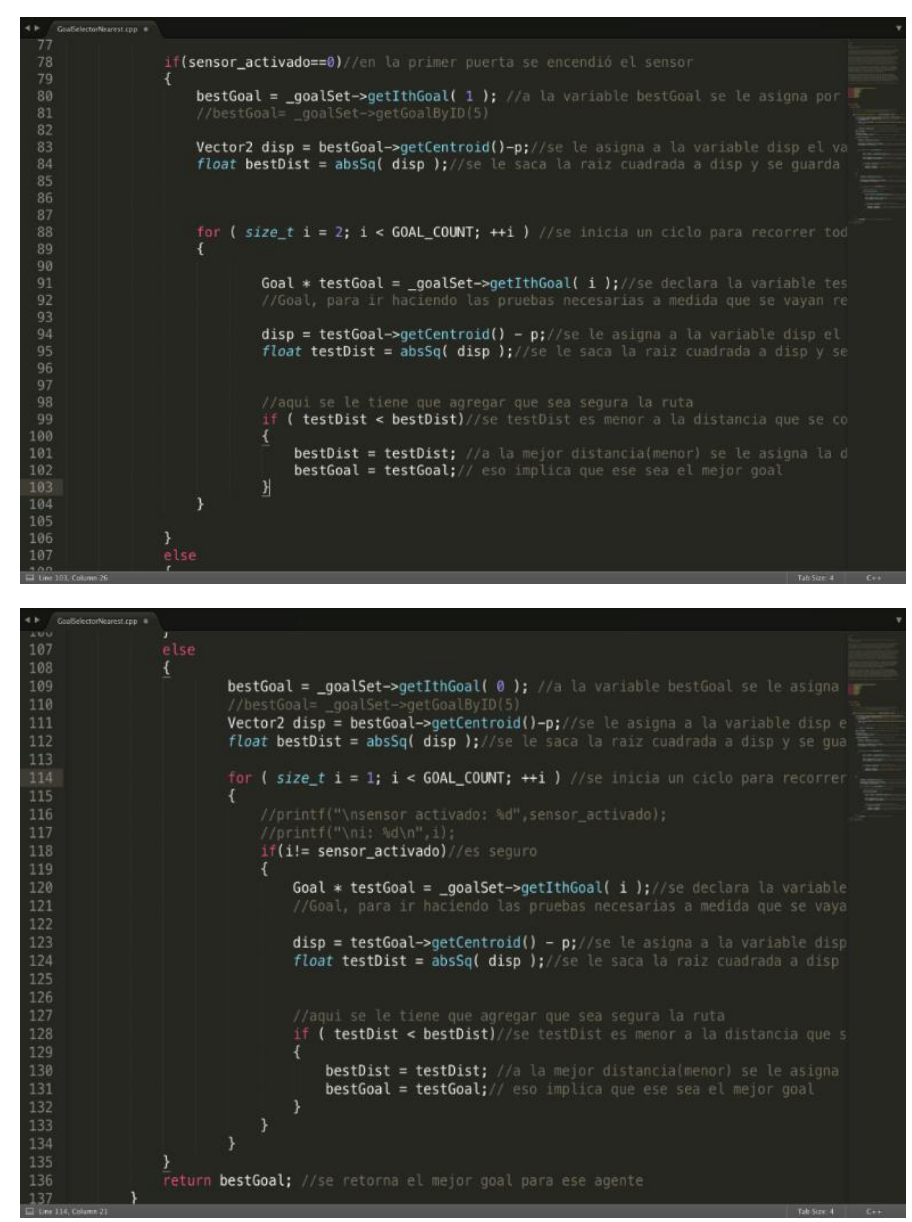

Fig. 1. Shows a variant of the Dijkstra's algorithm, which is written in $\mathrm{C}++$, finding the shortest and secure path function. 


\subsection{Mathematical Model of Spread of Fire inside a Passenger Vessel}

The development of a mathematical model for predicting rate of fire spread and intensity to a wide range of fire inside a passenger vessel is presented from the conceptual stage through evaluation, variables as Oxidizing, fuel, wind velocity, relative humidity, slope, and temperature or activation energy were all recognized as producing important effects of the spread of fire. These factors were studied and correlated to some form of fire behavior.

1. Fuel: Any material layers to release energy when oxidized violently with heat release (Any flammable material).

2. Oxidizing 2- (oxidizing agent) is an agent that can oxidize a fuel and in doing this boils down to itself (in our case is oxygen, the air is composed of about $21 \%$ oxygen)

3. Temperature or activation energy: this is the amount of heat needed to start the combustion of a material.

REACTION FREES CHAIN: A process that allows for continuity and spread of fire giving off heat that is transmitted to fuel combustion and continuing as long as the supply of fuel and oxidizer is maintained.

$$
C R_{(t i)}=(F * H * O * T) * W V,
$$

$\mathrm{F} \rightarrow$ Fuel.

$\mathrm{O} \rightarrow$ oxidizer.

$\mathrm{T} \rightarrow$ Temperature.

$\mathrm{CR} \rightarrow$ Chain reaction.

$\mathrm{H} \rightarrow$ moisture.

$\mathrm{WV} \rightarrow$ wind velocity.

This equation will be used to go to adding or subtracting the coordinate values of the state to be a final this way when an agent is reached by the fire area die example:

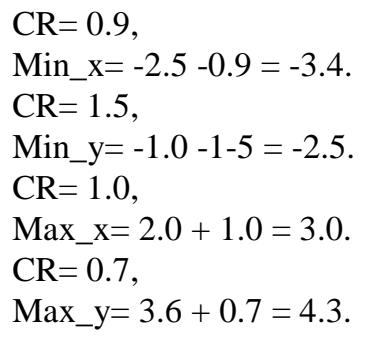

\section{Problem Statement}

The overall problem is addressed, is better problem addressed in this dissertation is to develop a methodology for emergency evacuation in the face of fire on a passenger's vessel 
planning that considers to help and management options of scape by choosing the shortest path for each passenger in order to facilitate the mobility of evacuees to the safety. The methodology is manifested in the development of a system of finding the shortest paths for each passenger. The objective of this research was to review current emergency evacuation plans and procedures for a passenger vessel. And also make recommendations for the use of advanced technologies where appropriate to improve evacuation during a sinister. The study did not consider an earthquake which are essential parts of a sinister emergency management system. This information could allow officials determine which areas need to be evacuated and when the call for mandatory evacuation is needed. Accurate tracking of a fire aboard reduces the chances of the decision-makers to make false alarms. Repeated false alarms will have a negative effect on the population as they start losing faith in the decisionmakers. Thus, false alarms should be avoided.

\section{$4 \quad$ Models of the Interfaces}

The following figures show the interface modules.

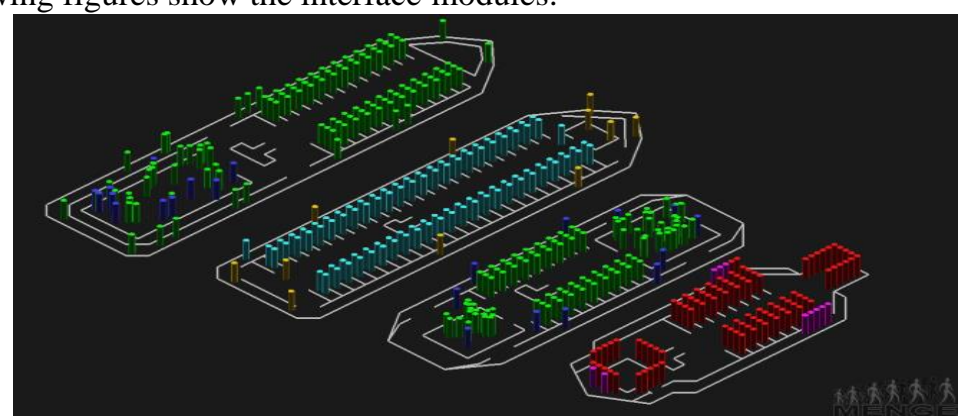

Fig. 2. Implementation in Menge: Main screen simulator contains the view of the 4 levels of the boat deck and the passengers for each level, using a simulation framework Menge.

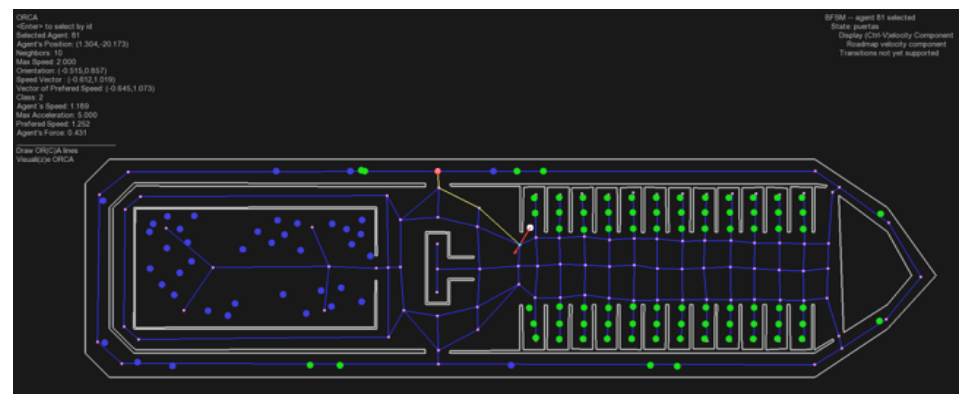

Fig. 3. Implementation in Menge: The screen shows the implantation of the system finding the shortest paths between departures in vessel decks for each passenger and the departures is free of danger. 


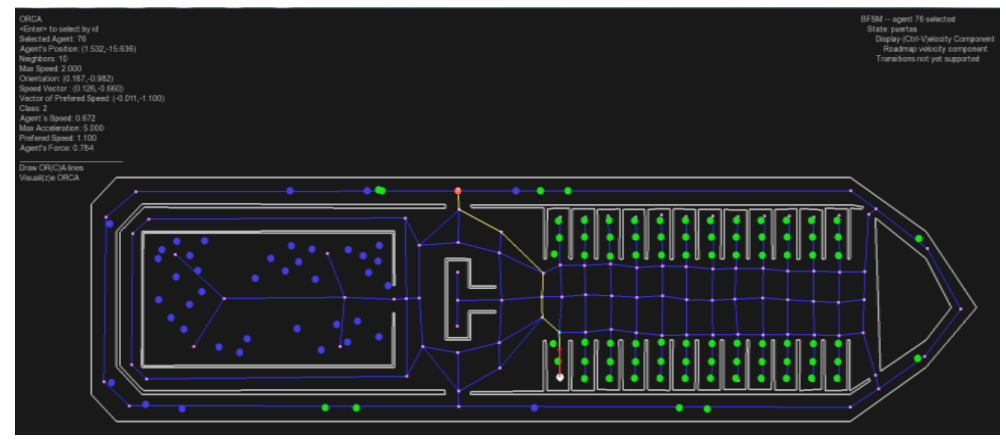

Fig. 4. Implementation in Menge: The screen shows the recalculation of the departures to the selected passenger, due to the state of danger occasioned in his appropriate departure.

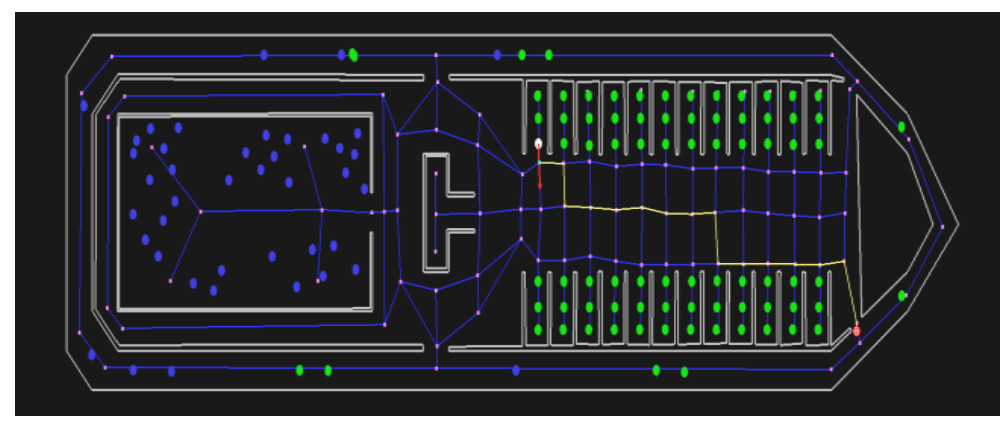

Fig. 5. Implementation in Menge: The screen shows how the selected passenger is obtaining the farthest route to his departure, due to the passenger is using a random evacuation route.

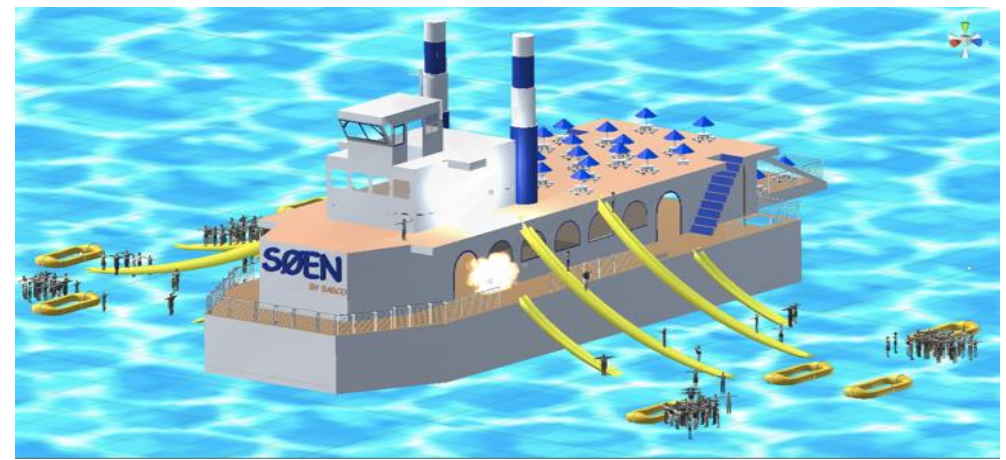

Fig. 6. Implementation in Unity, we can visualize these results in real time with the $3 \mathrm{D}$ viewer using the software unity. 


\section{Design of Experiments (DOE)}

It is worth mentioning that every simulation, the position of each passenger is the same. The total of passengers aboard in the vessel is of 443 passengers. Using the shortest path, achievement saves the 100 percent of the capacity crowd, in which they were made 25 simulations with 36 seconds on average to evacuate.

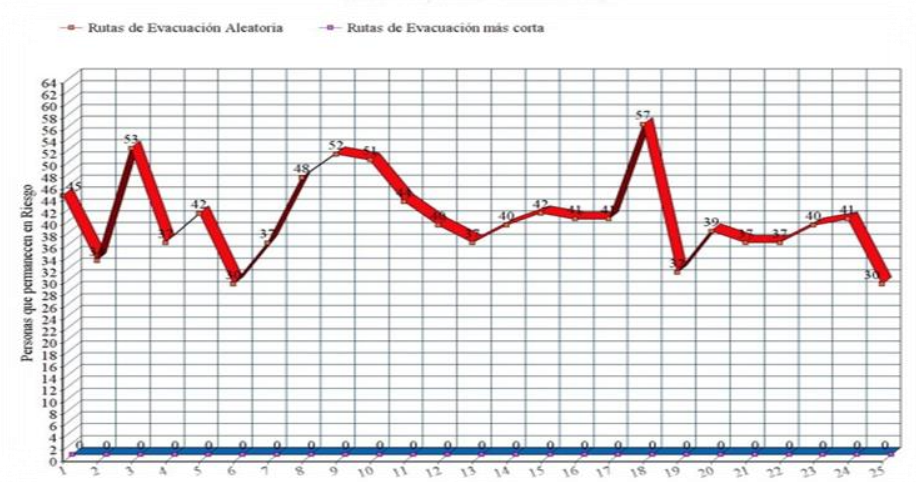

Fig. 7. Passenger flow at the vessel decks in the exercise and in the simulation counting ( $\mathrm{Y}$ axis: number of passengers who still staying in a state of danger and $X$ axis: Numbers of simulations. The result of the simulation selecting one departure of evacuation in a random way: red, the result of the simulation using the shortest and secure path system: Blue.

Afterwards were made 25 simulations in mode in which each agent selects one departure of evacuation in a random way in the same 36 seconds, in order to view the results in that way, it was found that on average of 39 passengers who were in the state of danger (in the inner part of the boat covers) which is equivalent to the 8.8 percent of the capacity crowd; in other words, is achieved only allocating the 91 percent of the capacity crowd into safety. Using that, every agent chooses their own evacuation path without knowing if it will lead them to risk or safety, while we were using the method of the shortest path of evacuation for each agent, is achieved to save the 100 percent of the capacity crowd.

With this we prove the following hypotheses: minimize through the use of software simulation the loss of lives in a sinister even who is the passenger. The percentage of passengers that were safe in comparison to using the system of the shortest path, indicated in the paper, shows that $100 \%$ of capacity crowd on the vessel is achieved to be safe while without the use of the system only $91 \%$ of capacity crowd were save.

\section{Conclusions and Future Research}

This research has analyzed the risks associated with collision, fire aboard and grounding of a passenger vessel and the importance of designing for safe, swift and efficient evacuation 
on board of a passenger vessel. Representing a serious threat to maritime safety and for the passenger vessel carrying a large number of people on board. This sinister have the potential to become devastating, and investigate possible events subsequent to an incident, sinking and capsizing, expected time to sink, etc.

In order to study fire aboard, grounding and collision scenarios, we have been developed models, to improve the evacuation performance. The research that we reported here sought to prove the following hypotheses: minimize through the use of software simulation the loss of lives in a sinister even who is the passenger, I mean the socioeconomic status and the socioeconomic class stratification. Unfortunately, due to socioeconomic diversity among the passengers, the layout of the ship, the evacuation is not as fluid as one would expect. These factors coupled together may have led directly impacting the survivability of a disaster. The research proposed intends on determining to what extent the socioeconomic factors played in the low mortality rate of a passenger vessel.

Those in charge of an emergency on a passenger ship believe that: panic is a natural occurrence in an emergency, sounding an alarm, such as a fire alarm, will cause panic, as a result of the above, the alarm should be delayed until absolutely necessary, the research also sought to prove that the above assumptions and beliefs are generally held as a result of media reporting of incidents rather than actual participation in emergencies. Finally, some risk control options are proposed that might reduce the risk of collision, fire aboard and grounding of passenger vessel by implementing a system of finding the shortest paths for each passenger. The information obtained from the simulations and the research will however have to be evaluated against general knowledge about crowd behavior and specific knowledge about in a situation behavior in emergencies and finding the shortest paths between exits in vessel decks. Our evaluation has shown a significant improvement in the evacuation rates when using a system of finding the shortest paths between exits in vessel decks for each passenger using a bracelet. Only a relatively small percentage of agents with good skills yields the best evacuation rates. We can visualize these results in real time with either our simple 2D using the software Menge or 3D.

Acknowledgments. This study has been supported by the Barcelona Supercomputing Center, located at the Torre Girona chapel and the National Prevention Center of disasters (CENAPRED) , would like to thank Dr. Alberto Ochoa Zezzati, Research Professor, Autonomous university of Juarez city, who inspired us to the research.

\section{References}

1. Arnskiv, M. M.: Waydinfing Systems, A proposal for new wayfinding system of passenger ferries. MEP desing report, Lyngby: DML (1999)

2. Emergency Management Australia: Flood Warning: An Australian Guide. Mount Macedon, Victoria (1995)

3. Human factors in ship design \& operation II. RINA, London (2002) 
4. May, M.: Wayfinding, Passenger behavior and maritime emergencies sings. In: Dick de Waard (ed.), Proceedings of the annual Meetings of the human factors and ergonomics society Europe, Chapter November 7-9, Torino (2001)

5. May, M., Jorgensen, H. D., Ostergaard, E.: Panning of mustering on board $\mathrm{m} / \mathrm{f}$ Kronprins Frederik. MEPdesing Report, Lyngby: DMI \& Scandlines (2000)

6. Passini, P.: Wayfinding in architecture. Van Nostrand, New York (1992)

7. Perry, R, W.: A model of evacuation compliance behavior. In: Dynes, R. R \& Tierney K. J (eds.), Disasters, collective behavior and social organization, University of Delaware Press, Newark (1994)

8. Withington, D.: Localisable, Alarms. In: Stanton, N. A. \& Edworthy, J. (eds.), Human factors in auditory Warnings, Ashgate, Aldershot (1999)

9. Withington, D.: Use of Directional sound for ship Evacuation. In: 8th Word conference of Emergency Management (TIEMS 2001), Oslo, pp. 19-21 (2001)

10. Chamero, J.: Dijkstra's Algorithm Discrete Structures \& Algorithms. (2006)

11. Helbing, D., et al.: Self-organized pedestrian crowd dynamics: Experiments, simulations, and design solutions. Transportation science, Vol. 39, No. 1, pp. 1-24 (2005)

12. Helbing, D., Molnar, P.: Social force model for pedestrian dynamics. Physical review, Vol. 51, No. 5 (1995)

13. Helbing, D., et al.: Simulation of pedestrian crowds in normal and evacuation situations. Pedestrian and evacuation dynamics, Vol. 21, No. 2, pp. 21-58 (2002) 